\title{
Antinociceptive effects of hyaluronic acid on monoiodoacetate-induced ankle osteoarthritis in rats
}

\author{
Shunsuke Jimbo' \\ Yoshinori Terashima ${ }^{1,2}$ \\ Atsushi Teramoto' \\ Tsuneo Takebayashi ${ }^{3}$ \\ Izaya Ogon' \\ Kota Watanabe 4 \\ Tatsuya Sato ${ }^{2,5}$ \\ Nobutoshi Ichise ${ }^{2}$ \\ Noritsugu Tohse ${ }^{2}$ \\ Toshihiko Yamashita' \\ 'Department of Orthopaedic surgery, \\ Sapporo Medical University School \\ of Medicine, Sapporo 060-8556, \\ Japan; ${ }^{2}$ Department of Cellular \\ Physiology and Signal Transduction, \\ Sapporo Medical University School of \\ Medicine, Sapporo 060-8556, Japan; \\ ${ }^{3}$ Sapporo Maruyama Orthopaedic \\ Hospital, Sapporo 060-0007, Japan; \\ ${ }^{4}$ Department of Second Division \\ of Physical Therapy, Sapporo \\ Medical University School of Health \\ Sciences, Sapporo 060-8556, Japan; \\ ${ }^{5}$ Department of Cardiovascular, \\ Renal and Metabolic Medicine, \\ Sapporo Medical University School of \\ Medicine, Sapporo 060-8556, Japan
}

Correspondence: Shunsuke Jimbo Department of Orthopaedic surgery, Sapporo Medical University School of Medicine, South-I, West-16, Chuo-ku, Sapporo 060-8556, Japan

Tel $+8 \mid$ II $61|2| I \mid$

Fax +8I II 62I 8059

Email s-jimbo@sapmed.ac.jp
This article was published in the following Dove Medical Press journal: Journal of Pain Research

\begin{abstract}
Purpose: Ankle osteoarthritis (OA) causes significant pain and debilitation; yet, its underlying mechanisms remain unclear. Clinically, hyaluronic acid (HA) is widely used to treat OA. The present study aimed to investigate the roles of HA in pain-related behavior, joint function, swelling, and pathological changes in cartilage in a rat model of monoiodoacetate (MIA)induced ankle OA.
\end{abstract}

Materials and methods: Male Sprague Dawley rats were assigned to three experimental groups as follows: 1) MIA rats injected with $1 \mathrm{mg}$ MIA in the right tibiotarsal joint for two consecutive days; 2) sham rats injected with saline instead of MIA; and 3) MIA-HA rats injected with $\mathrm{HA}$ in the tibiotarsal joint at 7, 14, and 21 days after MIA injection. Joint swelling, range of motion (ROM), and pain-related behavior were evaluated 1 day before and on the 7 th, 14 th, 21 st, and 28th day after MIA or saline injection. Pathological changes in the ankle joint were assessed 28 days after MIA or saline injection.

Results: No significant difference in the degree of ankle swelling or ROM reduction was observed between MIA rats and MIA-HA rats. However, compared with those in MIA rats, mechanical and thermal hypersensitivity was significantly reduced and stride length significantly improved in MIA-HA rats. Histologic analysis revealed that cartilage degeneration was significantly suppressed in MIA-HA rats compared with that in MIA rats, reflecting the chondroprotective effects of HA.

Conclusion: HA improved pain-related behavior and stride length and suppressed MIA-induced cartilage degeneration. HA may thus inhibit OA progression and suppress peripheral and/or central sensitization.

Keywords: ankle osteoarthritis, monoiodoacetate, osteoarthritis pain, hyaluronic acid

\section{Introduction}

Osteoarthritis (OA) is one of the most prevalent and debilitating joint diseases and is associated with reduced quality of life and increased health care costs. OA occurrence is attributed to cartilage degeneration, fibrillation, erosion, and osteophyte formation and is characterized by chronic pain and loss of joint function in the majority of patients. ${ }^{1}$ While the prevalence of knee OA is $\sim 15 \%-20 \%$ in the adult population, ankle OA accounts for $\sim 1 \%$ of all cases. ${ }^{2,3}$ Ankle OA tends to occur in older adult populations and displays gradual progression; however, patients with ankle OA tend to be younger than patients with hip OA and also experience longstanding chronic pain that affects their quality of life. ${ }^{4}$ Further, with increases in the aging population and sports-related injuries, the incidence of ankle OA has been increasing. ${ }^{5}$ The progression to end-stage ankle OA has been reported to be $\sim 10-20$ years after the initial injury and tends to occur in the middle age among most patients. ${ }^{6}$ Therefore, we should not overlook the

Journal of Pain Research 2019:12 191-200

I91 (c) (i) (5) 2019 jimbo et al. This work is published and licensed by Dove Medical Press Limited. The full terms of this license are available at https://www.dovepress.com/terms. php and incorporate the Creative Commons Attribution - Non Commercial (unported, v3.0) License (http:///creativecommons.org/licenses//by-nc/3.0/). By accessing the work you hereby accept the Terms. Non-commercial uses of the work are permitted without any further permission from Dove Medical Press Limited, provided the work is properly attributed. For permission for commercial use of this work, please see paragraphs 4.2 and 5 of our Terms (https://www.dovepress.com/terms.php) 
burden and the socioeconomic impact of ankle OA. ${ }^{7}$ Currently available medical therapies for OA, which include traditional analgesics and non-steroidal anti-inflammatory drugs (NSAIDs), are ineffective in altering or slowing disease progression, but rather alleviate symptoms by reducing pain and improving joint mobility. Further, their chronic use has been restricted by their deleterious side effects, and surgical interventions such as total ankle replacement and arthrodesis are eventually required. ${ }^{8,9}$

In 1997, the US Food and Drug Administration approved intra-articular injections of hyaluronic acid (HA) for the treatment of OA. ${ }^{9}$ Intra-articular HA injections can restore viscoelasticity by replacing the dysfunctional synovial fluid, which, due to the modulation of early inflammatory responses, might also exert an analgesic effect on the area. ${ }^{10} \mathrm{HA}$ protects collagen fibrils, cells of the articular surface, synovial tissue, capsule, and ligament from mechanical damage. Moreover, intra-articular HA knee injections have been shown to have a chondroprotective effect in an early knee OA rat model. ${ }^{11}$ HA has been widely used in the management of knee OA, and the efficacy of this treatment has been confirmed in several meta-analyses. ${ }^{9,12-14}$

In a rat knee OA model, the subchondral bone was found to be associated with inflammatory pain and expression of pain-related receptors. ${ }^{15}$ Although OA pain may be correlated with peripheral and central sensitization, the mechanisms involved in the pain and pathophysiology remain unclear. ${ }^{16}$ There have been no fundamental studies investigating the antinociceptive effect of intra-articular HA injection in ankle OA.

The aging-based model of ankle OA has the distinct disadvantage of prolonged delay and is associated with slight cartilage denaturation. The surgically induced model of ankle OA is created by cutting ligaments around the ankle joint; ${ }^{17}$ however, differences in surgical expertise can affect the degree of cartilage denaturation. The ankle fracture model also relies on experimental skill. ${ }^{18}$ Consequently, these models have reproducibility issues. We have previously reported the utility of the rat model of monoiodoacetate (MIA)-induced ankle OA, which can be easily reproduced. ${ }^{19}$

The present study aimed to evaluate the efficacy of intraarticular ankle injection of HA, a previously unreported strategy, in a rat model of MIA-induced ankle OA.

\section{Materials and methods Hyaluronic acid}

We used ARTZ-Dispo HA (weight-average molecular weight [MW], 60-120 kDa; viscosity-average MW, 1,650 kDa; Seikagaku, Tokyo, Japan).

\section{Animal preparation and intra-articular injection}

All experimental procedures in this study were performed according to the Ethical Guidelines of the International Association for the Study of Pain and were approved by the Animal Care and Use Committee of Sapporo Medical University School of Medicine (Sapporo, Japan). Efforts were made to minimize the number of animals used.

We used 60 male Sprague Dawley rats (3 weeks old; body weight, $46-58 \mathrm{~g}$ ). The rats were assigned to three experimental groups of 20 rats each (including 15 rats for behavioral study and 5 rats for pathological study). Rats in the MIA group were injected with MIA $(1 \mathrm{mg} / 30 \mu \mathrm{L})$ in the tibiotarsal joint of the right hind paw for two consecutive days, as previously described. ${ }^{19}$ Rats in the sham group were injected with saline $(30 \mu \mathrm{L})$. Both MIA and sham rats were injected with saline $(30 \mu \mathrm{L})$ in the tibiotarsal joint of the right hind paw at 7, 14, and 21 days after MIA/saline injection. Rats in the MIA-HA group were injected with HA $(30 \mu \mathrm{L})$ into the tibiotarsal joint of the right hind paw at 7, 14, and 21 days after MIA injection.

\section{Ankle measurement}

At all of the indicated time points, we examined differences in the transverse and anteroposterior diameter and range of motion (ROM) in the affected and unaffected ankles. Ankle diameters were measured using digital calipers at the ankle neutral position. Each diameter was measured three times at each time point.

ROM of each ankle was measured using a protractor. The dorsiflexion angle was subtracted from the plantarflexion angle, and the arcs of the right and left ankles were calculated. The differences between right and left ankle arcs were then compared at all time points.

Walking stride length on an acrylic board $(7 \times 150 \times 12$ $\mathrm{cm})$ was also measured at all time points. Stride length was measured three times using an iPad Air 2 device (Apple, Cupertino, CA, USA), and the average difference between the affected and unaffected sides was calculated.

\section{Behavioral analysis}

Pain-related behavior was assessed by observing the mechanical and thermal hind paw withdrawal responses before injection (pre-MIA/saline) and at 7, 14, 21, and 28 days after MIA/saline injection. For mechanical allodynia assessment of mechanical withdrawal responses, each rat was placed in a Plexiglas chamber $(18 \times 25 \times 18 \mathrm{~cm}$; IITC Life Science Inc., Woodland Hills, CA, USA) above a wire mesh floor, allowing full access to the area for at least 20 minutes before the test. 
Next, mechanical withdrawal responses to von Frey filament stimuli (2 and 4 g, Semmes Weinstein Monofilaments; North Coast Medical Inc., San Jose, CA, USA) were evaluated at the time points mentioned above, as previously described. ${ }^{20,21}$ Both the right and left hind paws were stimulated 30 times with a minimum interval of 10 minutes between sessions. The mechanical withdrawal frequency was recorded by subtracting the number of responses on the left side from that on the right side.

The thermal paw withdrawal response was measured by using a radiant heat source (Tail Flick Analgesia Meter; IITC Life Science Inc.) to evaluate hyperalgesia at all of the time points stated above. Each rat was placed in a Plexiglas chamber on a glass platform and allowed to acclimatize for at least 20 minutes before the test. The thermal withdrawal response was evaluated as the latency of hind paw withdrawal in response to thermal stimulation delivered using a radiant source that had been moved beneath the portion of the hind paw that was flushed against the glass. Each hind paw was tested five times, and the mean withdrawal latency was calculated. The thermal paw withdrawal latency was defined as asymmetrical left-right latency.

\section{Histologic assessments}

Rats were sacrificed 28 days after MIA/saline injection for histologic assessments. The right ankle joints were dissected, fixed in $10 \%$ formalin, and embedded in paraffin. Sections were prepared and stained with H\&E or safranin-O fast green and observed under a microscope. We observed the histopathologic changes on the distal medial/lateral tibial surface and the proximal medial/lateral talus surface of the right hind paw tibiotarsal joint. The Osteoarthritis Research Society International (OARSI) OA cartilage histopathology assessment system was used to assess the severity of articular cartilage damage. ${ }^{22}$ In this system, the grade of damage from 0 (normal) to 6 (severe) is defined as the progression of OA into the cartilage, and the stage of damage is defined as the horizontal extent of cartilage involvement from 0 (normal) to 4 (large). The final score is the combined value of grade and stage (score range of 0-24).

\section{Statistical analysis}

All numerical data are expressed as means and standard errors of the means. All data were analyzed using one-way repeated measures ANOVA and the Tukey-Kramer test for comparisons among the three experimental groups. $P<0.05$ was considered statistically significant.

\section{Results}

\section{Changes in ankle appearance}

When compared with sham rats, MIA and MIA-HA rats had significant swelling in the anteroposterior and transverse diameters of the right ankle at all four post-injection time points (Figure 1). No significant differences in ankle anteroposterior or transverse diameter were observed between MIA and MIA-HA rats at any post-injection time point. Therefore, HA injection produced no significant effects on ankle appearance.

Compared with the sham rats, a reduction in ROM was observed from 7 days after MIA/saline injection in both MIA and MIA-HA rats (Figure 2). In the sham rats, ROM was similar on both sides. Further, the ROM on the affected side was similar in both MIA-HA and MIA rats, indicating that HA did not improve ankle ROM.

\section{Evaluation of pain-related behavior}

The MIA and MIA-HA rats exhibited a significantly shorter walking stride than the sham rats at 7 days after MIA injection (Figure 3). However, only the MIA rats had a significantly shorter stride than the sham rats at 14, 21, and 28 days after $\mathrm{MIA} /$ saline injection. Further, the MIA rats also had a significantly shorter stride than the MIA-HA rats at 14, 21, and 28 days after MIA injection. No differences between MIA-HA rats and sham rats were observed at 14,21, and 28 days after injection. HA injections improved the stride length 14 days after MIA injection.

Compared with that in sham rats, the mechanical response frequency increased in both the MIA and MIA$\mathrm{HA}$ rats at 7 days after MIA/saline injection. We observed a significant increase in the response frequency of the ipsilateral hind paw to the 2 and $4 \mathrm{~g}$ filament in only MIA rats at 14, 21, and 28 days after MIA injection (Figure 4). Allodynia did not occur 14 days after MIA injection or thereafter in the MIA-HA rats.

Further, we observed significantly shorter thermal latency in the MIA rat hind paws than in the sham and MIA-HA rat hind paws 14 days after MIA/saline injection (Figure 5). In the MIA rats, hyperalgesia was seen 14 days after MIA injection, while MIA-HA rats did not exhibit hyperalgesia at any of the four post-injection time points.

\section{Histologic changes}

We observed histologic changes such as chondrocyte disorganization, erosion, surface fibrillation, and subchondral bone exposure in both MIA and MIA-HA rats 28 days after MIA injection. In the MIA rats, joint space narrowing and 
A

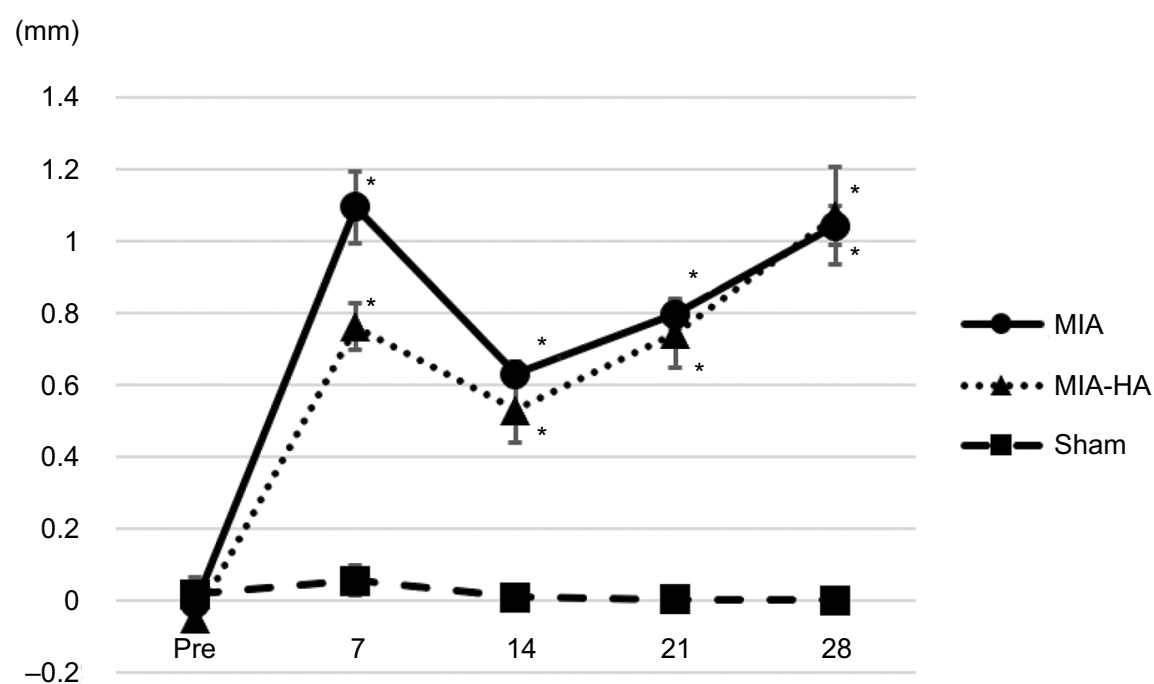

Time after surgery (days)

B

(mm)

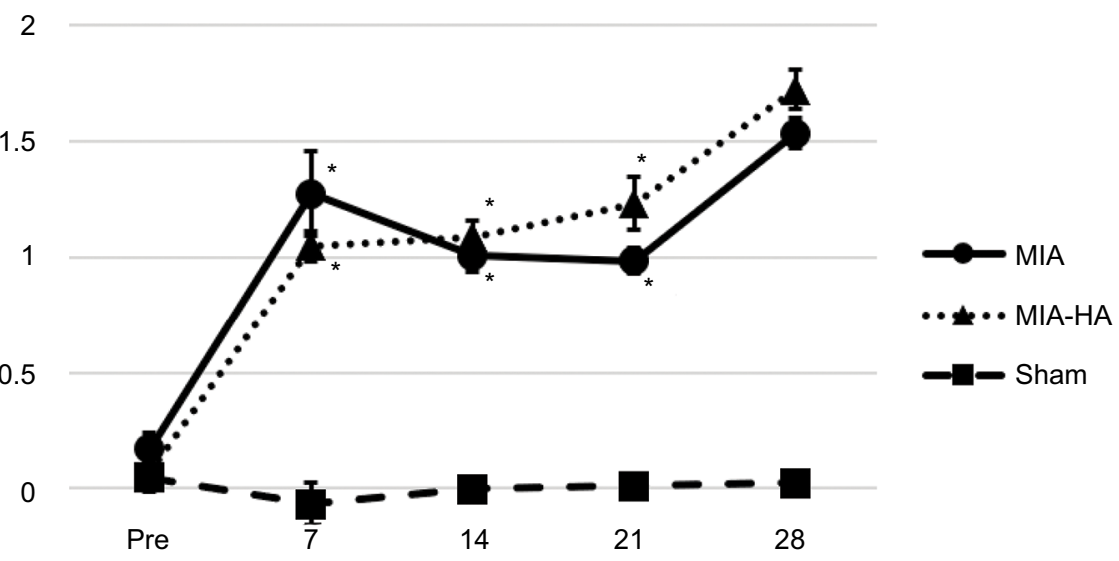

$-0.5$

Time after surgery (days)

Figure I Changes in ankle swelling.

Notes: The anteroposterior (A) and transverse (B) diameters of the ankle were elevated in the MIA group. When comparing MIA and MIA-HA rats, no significant differences in anteroposterior or transverse ankle diameter were observed at any post-injection time point. Data are shown as the mean \pm standard errors of the mean. ${ }^{*} P<0.05$ : significant difference compared to sham rats.

Abbreviations: HA, hyaluronic acid; MIA, monoiodoacetate.

cartilage denaturation were observed beginning 7 days after MIA injection (Figure 6). These changes progressed further 14 days after MIA injection. Collapse of the talus was observed 21 days after MIA injection. Histologic denaturation including chondrocyte disorganization, erosion, cartilage surface fibrillation, and subchondral bone exposure was observed in both the MIA and MIA-HA rats 28 days after MIA injection. However, the joint space was better preserved and cartilage denaturation was milder in the MIA-HA rats than in the MIA rats.

To evaluate these changes quantitatively, we utilized the OARSI OA cartilage histopathology assessment system (Table 1). MIA rats had a significantly higher OARSI grade than MIA-HA and sham rats at all sites. MIA-HA rats showed a significantly higher OARSI grade than sham rats in only the medial tibia. Both the MIA and MIA-HA rats had sig- 


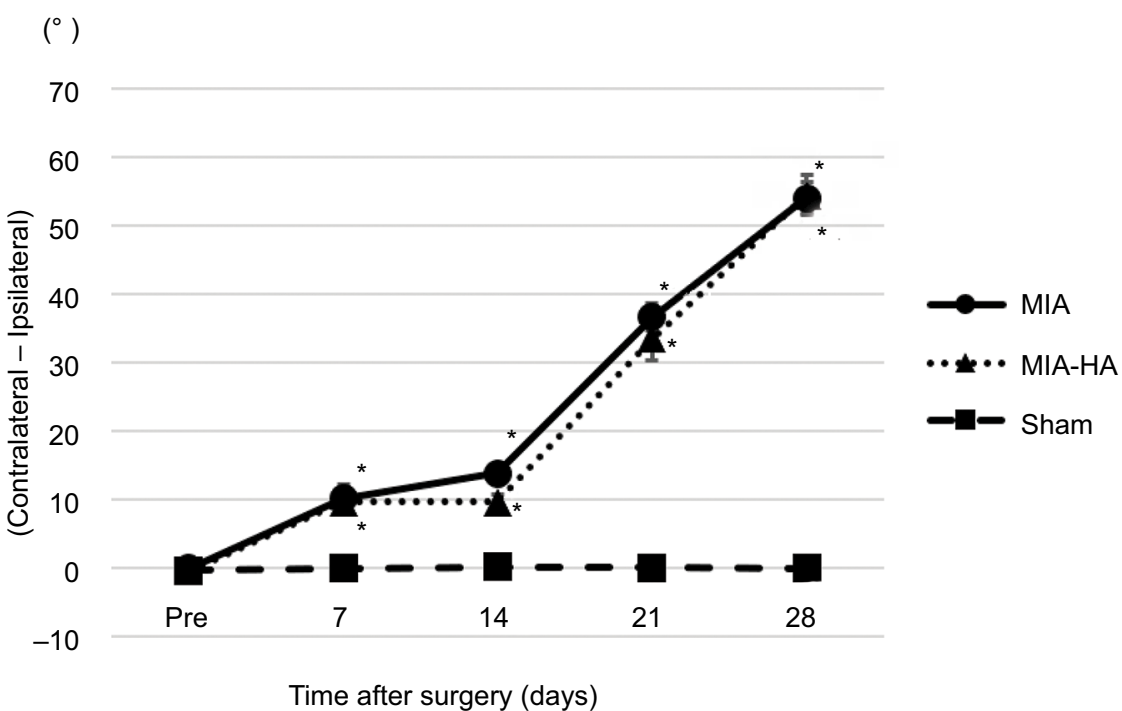

Figure 2 The range of motion of the ankle (arc of the affected side).

Notes: We subtracted the dorsiflexion angle from the plantarflexion angle and calculated the arc on the right and left ankles. Differences between right and left ankle arcs were then compared. No significant differences between MIA and MIA-HA groups were observed. ${ }^{*} P<0.05$ : significant difference compared to sham rats. Abbreviations: HA, hyaluronic acid; MIA, monoiodoacetate.

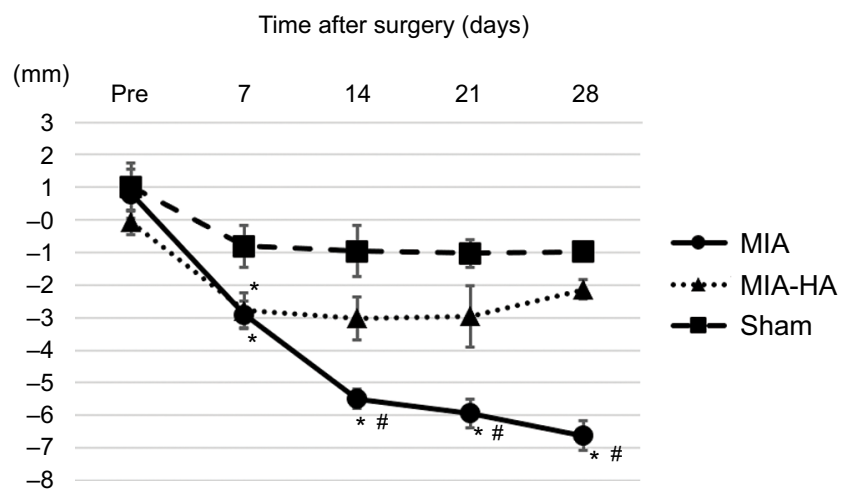

Figure 3 Change of stride length.

Notes: Compared with sham rats, MIA and MIA-HA rats exhibited a significantly shorter walking stride on the seventh day following MIA/saline injection. Only MIA rats had a significantly shorter stride than sham rats at 14, 21 , and 28 days after MIA saline injection. MIA rats also had a significantly shorter stride than MIA-HA rats at 14,21 , and 28 days after MIA injection. $* P<0.05$ : significant difference compared to sham rats. ${ }^{\#}<0.05$ : significant difference compared to MIA-HA rats.

Abbreviations: HA, hyaluronic acid; MIA, monoiodoacetate.

nificantly higher OARSI stages than sham rats at all sites. However, no significant difference in OARSI stage was found between MIA and MIA-HA rats at any site.

The OARSI score is the final combined value of grade and stage. MIA rats showed a significantly higher OARSI score than MIA-HA and sham rats at all sites. There was no significant difference between MIA-HA and sham rats at any site.

\section{Discussion}

OA is one of the most prevalent and debilitating joint diseases and is associated with reduced quality of life and increased health care costs. OA causes the loss of articular cartilage, damage to all joint structures including adjacent supporting connective tissue elements, subchondral bone changes, varying degrees of osteophyte formation, and synovitis. ${ }^{1}$ Recommendations for the management of OA, especially knee OA, have been issued by the European League Against Rheumatism, the European Society for Clinical and Economic Aspects of Osteoporosis and Osteoarthritis, the American College of Rheumatology, and the OARSI. ${ }^{23-28}$ Current treatment options include simple analgesics, NSAIDs, intraarticular HA or corticosteroid injections, orthotics, physical therapy, and surgical interventions. In case of contraindications to NSAIDs, severe symptoms, or if the patient is still symptomatic despite NSAID use, intra-articular treatment may be applied..$^{29}$ Intra-articular HA injections are relatively safe, and compared with intra-articular corticosteroids, HA induces long-lasting pain control. ${ }^{14}$ In a meta-analysis, Chang et al reported that, compared to their pretreatment condition, intra-articular HA administration significantly reduced pain for patients with ankle OA. ${ }^{30}$ Although increased HA injection volume does not result in additional pain relief, a positive relationship between total number of HA injection doses and effect size was observed, and the use of multiple doses is likely more beneficial than a single injection. Witteveen et al reported that three weekly injections of HA provided greater pain relief than a single injection. ${ }^{31}$ Minimal relations existed between HA MW and treatment response. High-MW HA has a higher viscosity and is eliminated from the joints more slowly than lower-MW HA. ${ }^{31,32}$ 
A

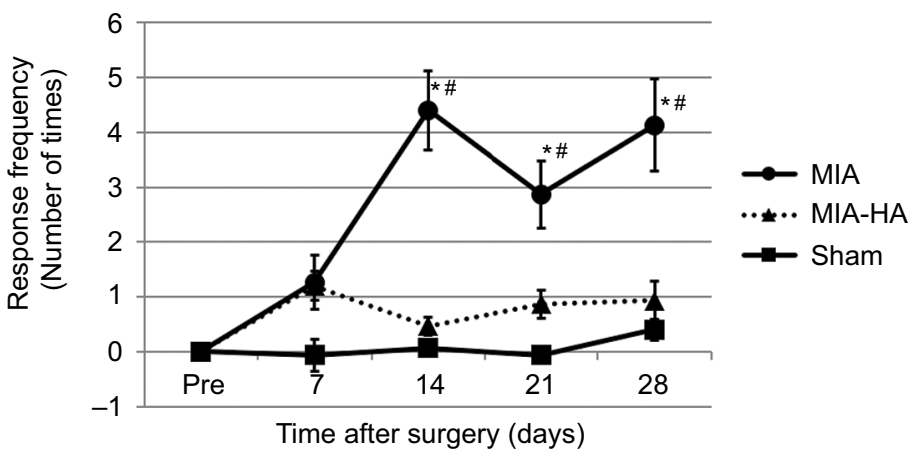

B

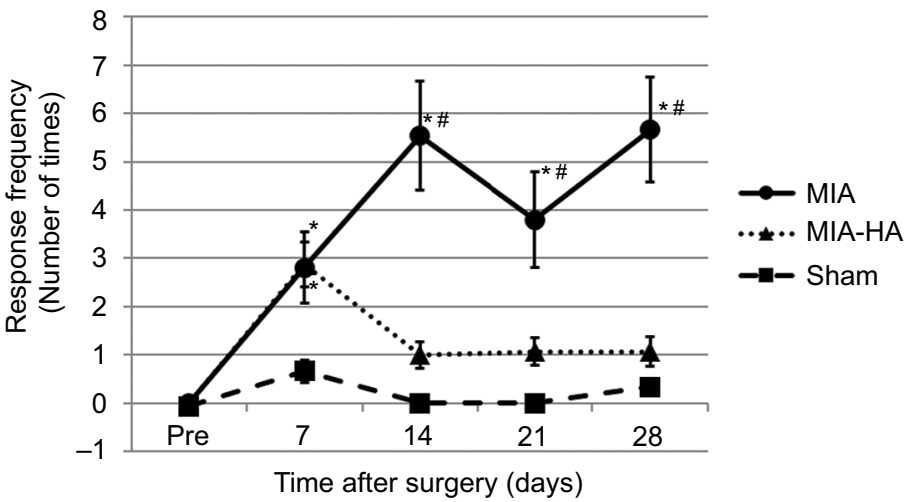

Figure 4 Assessment of mechanical hypersensitivity (allodynia).

Notes: A significant increase in the response frequency of the ipsilateral hind paw was only observed in MIA rats at I4, 21 , and 28 days after MIA injection. (A) von Frey 2 $g$ and (B) $4 \mathrm{~g}$ filaments. ${ }^{*} P<0.05$ : significant difference compared to sham rats. ${ }^{*} P<0.05$ : significant difference compared to MIA-HA rats.

Abbreviations: HA, hyaluronic acid; MIA, monoiodoacetate.

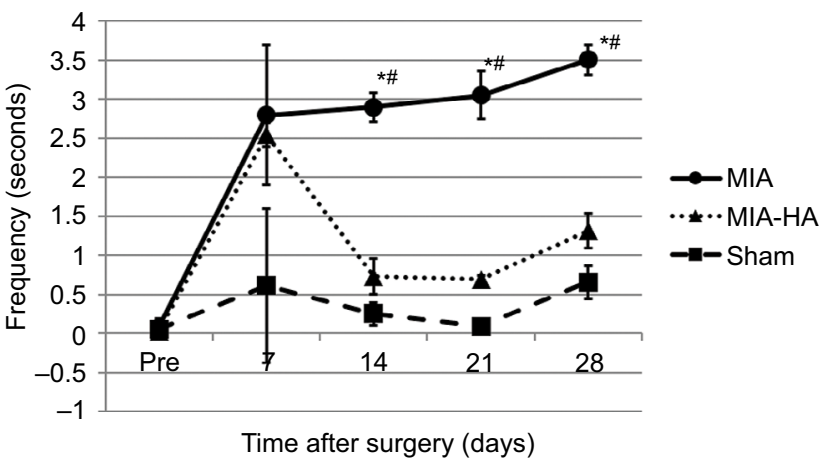

Figure 5 Assessment of thermal hyperalgesia.

Notes: In MIA rats, hyperalgesia was seen 14 days after MIA injection. MIA-HA rats did not exhibit hyperalgesia at any post-injection time point. ${ }^{*} P<0.05$ : significant difference compared to sham rats. ${ }^{*}<<0.05$ : significant difference compared to MIAHA rats.

Abbreviations: HA, hyaluronic acid; MIA, monoiodoacetate.

We previously reported a rat model of MIA-induced ankle OA. ${ }^{19}$ This model is useful in studying ankle OA because cartilage degeneration is induced efficiently and the model has high reproducibility. The present study demonstrated that intra-articular injection of HA suppressed hypersensitivity, thermal hyperalgesia, and stride length in a rat model of MIA-induced ankle OA. Progression of joint denaturation was also suppressed. On the other hand, intra-articular HA injection did not affect ankle swelling or ankle ROM.

Balazs and Denlinger reported that HA can modulate the early inflammatory response and has anti-inflammatory effects. ${ }^{12}$ The structural breakdown of proteoglycans (PGs) and collagen is considered to be the result of increased chondrocyte catabolic activity. ${ }^{33} \mathrm{IL}-1 \beta$ is a cytokine involved in cartilage degradation. IL- $1 \beta$ causes chondrocytes to secrete neutral matix metalloproteinases (MMPs) and nitric oxide (NO). NO is a highly reactive and cytotoxic free radical that has been implicated in tissue injury, inhibition of matrix synthesis, and activation of MMPs. HA protects chondrocytes from the effects of IL-1 $\beta$, decreases NO levels, and facilitates the restoration of $\mathrm{PG} .^{34-36}$

In the present study, no improvement in ankle swelling was observed. This may be due to chronic MIA-induced inflammation. In addition, there is a possibility that ROM improvements were not seen because ankle swelling persisted. On the other hand, stride length was improved in HA 
A

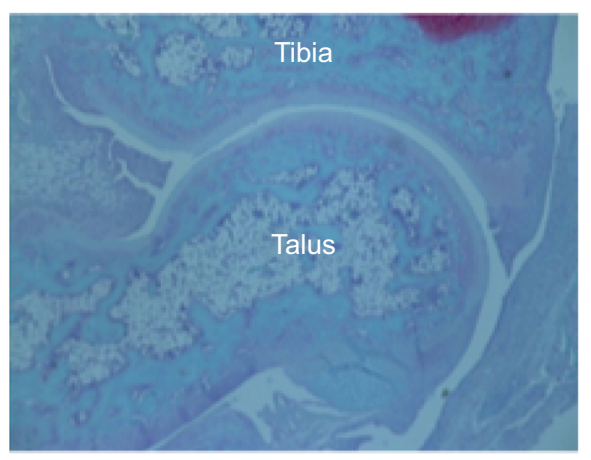

C

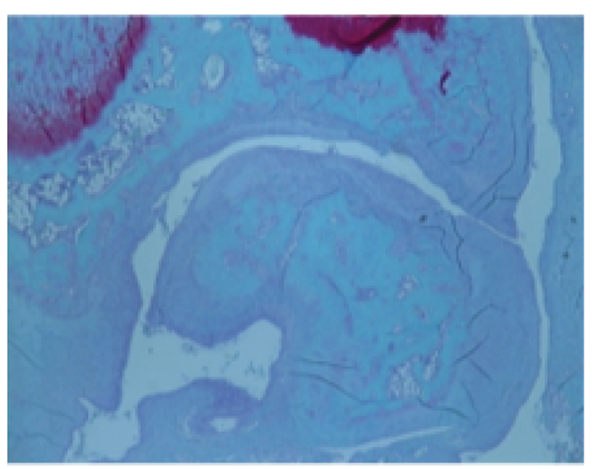

E

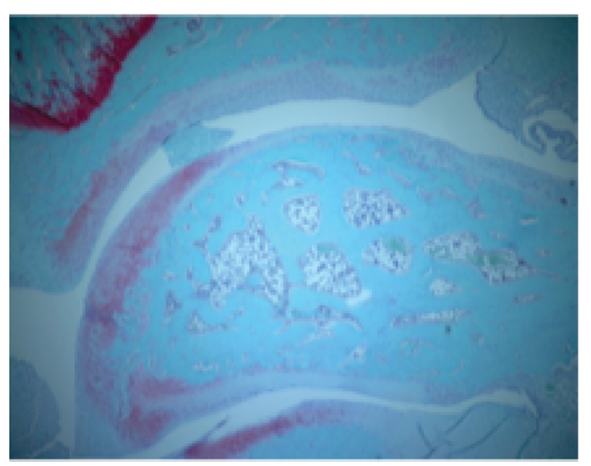

B

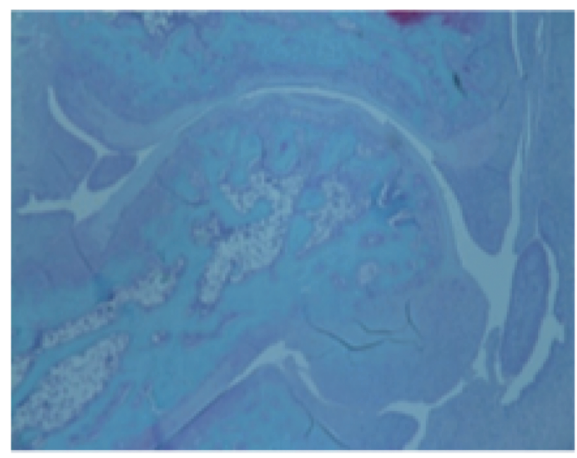

D

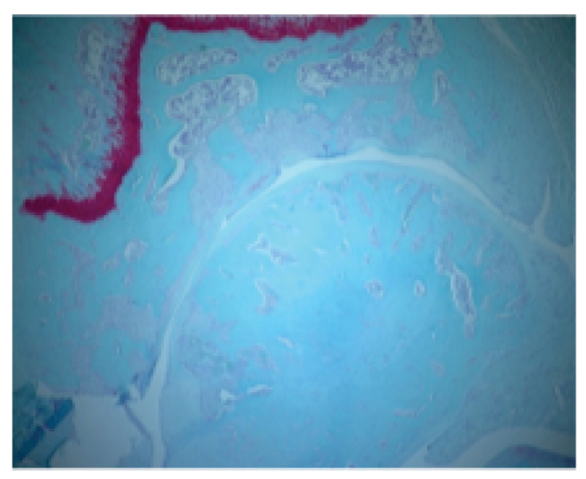

$\mathbf{F}$

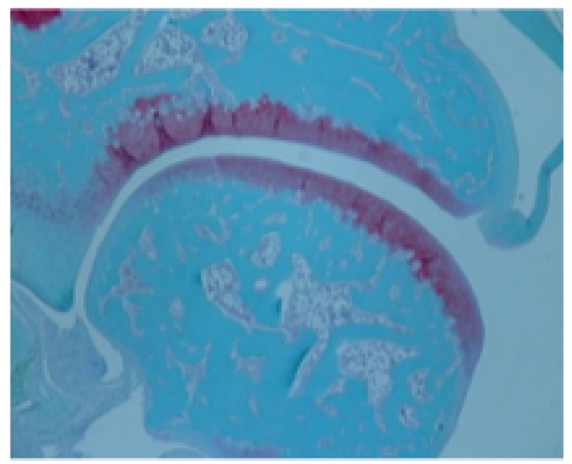

Figure 6 Histology of ankle joints.

Notes: (A-F) Histology of ankle joints obtained from each rat. (A) MIA rat 7 days after injection. (B) MIA rat 14 days after injection. (C) MIA rat 2 I days after injection. (D) MIA rat 28 days after injection. (E) MIA-HA rat 28 days after injection. (F) Sham rat 28 days after injection.

Abbreviations: HA, hyaluronic acid; MIA, monoiodoacetate.

rats, which we suspect occurred because intra-articular HA injections had an effect on pain relief. Peripheral sensitization of afferent nerve stimulus is typically seen after tissue damage or flare responses due to marked nociceptive neuropeptide production. This then results in increased sensitivity to heat and touch stimuli, referred to as primary hyperalgesia or primary allodynia if the stimulus was not painful prior to injury. ${ }^{37}$ Central sensitization represents an enhancement in the function of neurons and circuits in nociceptive pathways caused by increases in membrane excitability and synaptic efficacy. Central sensitization is also a manifestation of the remarkable plasticity of the somatosensory nervous system in response to activity, inflammation, and neural injury. ${ }^{38}$ Therefore, central sensitization produces pain hypersensitivity by changing the sensory response elicited by normal inputs, including those that usually evoke innocuous sensations. We used a von Frey test to evaluate allodynia and a plantar test for hyperalgesia. ${ }^{39,40}$ Thakur et al reported increased central sensitization and referred pain in a rat model of knee OA. ${ }^{41}$ They suggested that the existence of central changes in OA resembles neuropathic pain. Zhang et al reported that OA pain correlated with peripheral and central sensitization. ${ }^{16}$ 
Table I Histopathologic evaluation of safranin-O fast greenstained cartilage by OARSI cartilage OA histopathology grading system

\begin{tabular}{|l|l|l|l|}
\hline Area & MIA & MIA-HA & Sham \\
\hline Lateral tibia & & & \\
$\quad$ Grade & $5.0(0.3)^{*, \#}$ & $1.6(0.2)$ & $1.0(0)$ \\
Stage & $3.60 .4)^{*}$ & $2.6(0.2)^{*}$ & $0(0)$ \\
Score & $18.0(2.4)^{*, \#}$ & $4.2(0.8)$ & $0(0)$ \\
Medial tibia & & & \\
Grade & $4.6(0.2)^{*, \#}$ & $1.8(0.2)^{*}$ & $1.0(0)$ \\
Stage & $3.6(0.4)^{*}$ & $2.8(0.4)^{*}$ & $0(0)$ \\
Score & $16.4(1.8)^{*, \#}$ & $5.2(1.0)^{*}$ & $0(0)$ \\
Lateral talus & & & \\
Grade & $5.0(0.3)^{*, \#}$ & $1.8(0.5)$ & $1.0(0)$ \\
Stage & $3.4(0.2)^{*}$ & $2.2(0.6)^{*}$ & $0(0)$ \\
Score & $17.2(2.1)^{*, \#}$ & $5.0(2.3)$ & $0(0)$ \\
Medial talus & & & \\
Grade & $4.6(0.2)^{*, \#}$ & $2.2(0.6)$ & $1.6(0.2)$ \\
Stage & $3.8(0.2)^{*}$ & $2.6(0.2)^{*}$ & $1.2(0.5)$ \\
Score & $17.4(1.1)^{*, \#}$ & $5.0(2.3)$ & $2.4(1.0)$ \\
\hline
\end{tabular}

Notes: The OARSI score is the combined value of grade and stage (score range 0-24). Data represent the mean (standard error of the mean) for each group. $* P<0.05$ : significant difference compared to sham rats. ${ }^{\#}<<0.05$ : significant difference compared to MIA-HA rats.

Abbreviations: HA, hyaluronic acid; MIA, monoiodoacetate; OA, osteoarthritis; OARSI, Osteoarthritis Research Society International.

Ikeuchi et al examined the effect of intra-articular HA injection on pain in a rat model of knee OA. They reported that intra-articular HA injection reduced OA severity, decreased paw mechanical hyperalgesia, and attenuated OAassociated upregulation of calcitonin gene-related peptide (CGRP). However, no studies have investigated the effects of HA on pain relief in a model of ankle OA. ${ }^{42}$ In our study, significant mechanical hypersensitivity (ie, allodynia) was observed in the MIA rats, but not in the MIA-HA rats, 14 days after MIA injection. Similarly, we observed significant thermal hyperalgesia in the MIA rats, but not in the MIAHA rats 14 days after MIA injection. These observations suggested that HA suppressed peripheral and/or central sensitization. We speculated that nociceptive pain was alleviated due to intra-articular HA injection. Examination of biochemical and neurophysiological processes may provide an improved understanding of the pathogenesis of pain associated with ankle OA. The increases in c-Fos expression and the unregulated glutamate receptor subunit expression in the dorsal root ganglion and dorsal horn neurons are known to indicate the occurrence of peripheral and/or central sensitization. $^{43,44}$ Microglia and astrocyte activation in the dorsal horn neurons produces initiators and regulators of central sensitization pain including brain-derived neurotrophic factor and proinflammatory cytokines IL- $1 \beta$, tumor necrosis factor- $\alpha$, and IL-6, which modulate chronic pain. ${ }^{45}$ Further study is needed to assess pain associated with ankle OA to reflect a dominance of nociceptive, peripheral, and central pain mechanisms, respectively.

It is possible that allodynia is alleviated due to suppression of cartilage degeneration by intra-articular HA administration. Due to its resultant pain relief and joint functional improvements, viscosupplementation is used to treat $\mathrm{OA}$ worldwide. ${ }^{46} \mathrm{HA}$ is potentially effective as a chondroprotective agent due to alterations in catabolic and anabolic balance of the joint. ${ }^{47}$ Due to its poor oral bioavailability and risk of systemic toxicity, HA is a candidate for intra-articular use. ${ }^{9}$ Preliminary observations and evaluations of conventional approaches, such as intra-articular viscosupplemental administration of HA, have shown some convincing benefits and anti-inflammatory potential in both animal and human subjects. ${ }^{48-51}$

To the best of our knowledge, no study has investigated the effect of intra-articular HA administration in an ankle OA model. The articular cartilage of the ankle is more resistant to degeneration than that of the knee. ${ }^{52}$ Some studies have revealed several differences between the knee and ankle joint, including their structure, biomechanical properties, and chondrocyte phenotype. Biomechanical research has revealed that ankle cartilage has higher dynamic stiffness than knee cartilage. ${ }^{53}$ Moreover, each layer of articular cartilage in the ankle is thinner than that in the knee. ${ }^{54}$ In explant culture, ankle cartilage has been shown to be less responsive to catabolic stimulation than knee cartilage. ${ }^{55}$

In the present study, there was no significant difference in OARSI stage between MIA and MIA-HA rats; however, OARSI grade was significantly higher in MIA rats. Even in OARSI score, MIA rats displayed severe cartilage degeneration, but MIA-HA rats displayed an inhibition of degeneration. Based on these findings, we conclude that HA exerted a chondroprotective effect in a rat model of MIAinduced ankle OA. We infer that in addition to cartilage degeneration, sensitization was also suppressed by the antiinflammatory and viscosupplemental effects of HA.

\section{Limitations}

Limitations of our study should be considered. A major limitation is that we did not estimate the effects of different HA MWs and the frequency of administration. In addition, we did not immunohistochemically investigate the expression of pain-related receptors in the dorsal root ganglion and spinal cord. There are currently no immunohistochemical reports of this nature in a rat model of MIA-induced ankle OA. As such, further study is needed to assess pain associated with ankle OA to reflect a dominance of nociceptive, peripheral, and central pain mechanisms, respectively. 


\section{Conclusion}

The present study established the efficacy of a novel strategy, the intra-articular injection of HA in the ankle joint, in a rat model of MIA-induced ankle OA. We found that HA suppressed the pain-related behaviors associated with peripheral sensitization and/or central sensitization. Additionally, HA exerted a chondroprotective effect and inhibited the progression of ankle OA. Further studies are warranted to explore this strategy and identify molecules that alter pain transmission in the nervous system.

\section{Author contributions}

SJ and YT contributed to the study design, data analysis, interpretation of results, and writing of the manuscript. TT and TY contributed to the study design. AT, IO, KW, TS, NI, and NT contributed to the interpretation of results. All authors have read and approved the final manuscript. All authors contributed to data analysis, drafting and revising the article, gave final approval of the version to be published, and agree to be accountable for all aspects of the work.

\section{Disclosure}

The authors report no conflicts of interest in this work.

\section{References}

1. Loeser RF, Goldring SR, Scanzello CR, Goldring MB. Osteoarthritis: a disease of the joint as an organ. Arthritis Rheum. 2012;64(6):1697-1707.

2. Valderrabano V, Horisberger M, Russell I, Dougall H, Hintermann B. Etiology of ankle osteoarthritis. Clin Orthop Relat Res. 2009;467(7):1800-1806.

3. Brown TD, Johnston RC, Saltzman CL, Marsh JL, Buckwalter JA. Posttraumatic osteoarthritis: a first estimate of incidence, prevalence, and burden of disease. J Orthop Trauma. 2006;20(10):739-744.

4. Glazebrook M, Daniels T, Younger A, et al. Comparison of health-related quality of life between patients with end-stage ankle and hip arthrosis J Bone Joint Surg Am. 2008;90(3):499-505.

5. Kannus P, Palvanen M, Niemi S, Parkkari J, Järvinen M. Increasing number and incidence of low-trauma ankle fractures in elderly people: Finnish statistics during 1970-2000 and projections for the future. Bone. 2002;31(3):430-433.

6. Horisberger M, Valderrabano V, Hintermann B. Posttraumatic ankle osteoarthritis after ankle-related fractures. J Orthop Trauma. 2009;23(1):60-67.

7. Cushnaghan J, Dieppe P. Study of 500 patients with limb joint osteoarthritis. I. Analysis by age, sex, and distribution of symptomatic joint sites. Ann Rheum Dis. 1991;50(1):8-13.

8. Chevalier X. Intraarticular treatments for osteoarthritis: new perspectives. Curr Drug Targets. 2010;11(5):546-560.

9. Abramson S. Drug delivery in degenerative joint disease: where we are and where to go? Adv Drug Deliv Rev. 2006;58(2):125-127.

10. Lo GH, Lavalley M, Mcalindon T, Felson DT. Intra-articular hyaluronic acid in treatment of knee osteoarthritis: a meta-analysis. JAMA. 2003;290(23):3115-3121.

11. Şükür E, Talu C, Akman YE, Çirci E, Öztürkmen Y, Tüzüner T. Comparison of the chondroprotective effect of a novel hydrogel compound and traditional hyaluronate on rat cartilage in a papain-induced osteoarthritis model. Acta Orthop Traumatol Turc. 2016;50(4):458-463.
12. Balazs EA, Denlinger JL. Viscosupplementation: a new concept in the treatment of osteoarthritis. J Rheumatol Suppl. 1993;39:3-9.

13. Arrich J, Piribauer F, Mad P, Schmid D, Klaushofer K, Müllner M. Intraarticular hyaluronic acid for the treatment of osteoarthritis of the knee: systematic review and meta-analysis. CMAJ. 2005;172(8):1039-1043.

14. Bannuru RR, Natov NS, Obadan IE, Price LL, Schmid CH, Mcalindon TE. Therapeutic trajectory of hyaluronic acid versus corticosteroids in the treatment of knee osteoarthritis: a systematic review and metaanalysis. Arthritis Rheum. 2009;61(12):1704-1711.

15. Aso K, Izumi M, Sugimura N, Okanoue Y, Ushida T, Ikeuchi M. Nociceptive phenotype alterations of dorsal root ganglia neurons innervating the subchondral bone in osteoarthritic rat knee joints. Osteoarthritis Cartilage. 2016;24(9):1596-1603.

16. Zhang RX, Ren K, Dubner R. Osteoarthritis pain mechanisms: basic studies in animal models. Osteoarthritis Cartilage. 2013;21(9):1308-1315.

17. Chang SH, Yasui T, Taketomi S, et al. Comparison of mouse and human ankles and establishment of mouse ankle osteoarthritis models by surgically-induced instability. Osteoarthritis Cartilage. 2016;24(4):688-697.

18. Liang D, Sun J, Wei F, et al. Establishment of rat ankle post-traumatic osteoarthritis model induced by malleolus fracture. BMC Musculoskelet Disord. 2017;18(1):1821-1829.

19. Jimbo S, Terashima Y, Takebayashi T. Behavioral and histological characterization of ankle osteoarthritis model rat using monoiodoacetate. $J$ Arthritis. 2017.

20. Terashima Y, Kawamata M, Takebayashi T, Tanaka S, Tanimoto K, Yamashita T. Changes in synaptic transmission of substantia gelatinosa neurons in a rat model of lumbar radicular pain revealed by in vivo patch-clamp recording. Pain. 2011;152(5):1024-1032.

21. Miyakawa T, TerashimaY, Takebayashi T, et al. Transient receptor potential ankyrin 1 in spinal cord dorsal horn is involved in neuropathic pain in nerve root constriction rats. Mol Pain. 2014;10:1744-8069-10-58.

22. Pritzker KP, Gay S, Jimenez SA, et al. Osteoarthritis cartilage histopathology: grading and staging. Osteoarthritis Cartilage. 2006;14(1):13-29.

23. Jordan KM, Arden NK, Doherty M, et al. EULAR Recommendations 2003: an evidence based approach to the management of knee osteoarthritis: report of a task force of the Standing Committee for International Clinical Studies Including Therapeutic Trials (ESCISIT). Ann Rheum Dis. 2003;62(12):1145-1155.

24. Hochberg MC, Altman RD, April KT. American College of Rheumatology 2012 recommendations for the use of the hand, hip, and knee. Arthritis Care Res. 2012;64:465-474.

25. Zhang W, Moskowitz RW, Nuki G, et al. OARSI recommendations for the management of hip and knee osteoarthritis, part I: critical appraisal of existing treatment guidelines and systematic review of current research evidence. Osteoarthritis Cartilage. 2007;15(9):981-1000.

26. Zhang W, Moskowitz RW, Nuki G, et al. OARSI recommendations for the management of hip and knee osteoarthritis, part II: OARSI evidence-based, expert consensus guidelines. Osteoarthritis Cartilage. 2008;16(2):137-162.

27. Zhang W, Nuki G, Moskowitz RW, et al. OARSI recommendations for the management of hip and knee osteoarthritis: part III: changes in evidence following systematic cumulative update of research published through January 2009. Osteoarthritis Cartilage. 2010;18(4):476-499.

28. Mcalindon TE, Bannuru RR, Sullivan MC, et al. OARSI guidelines for the non-surgical management of knee osteoarthritis. Osteoarthritis Cartilage. 2014;22(3):363-388.

29. Bruyère $\mathrm{O}$, Cooper $\mathrm{C}$, Pelletier JP, et al. An algorithm recommendation for the management of knee osteoarthritis in Europe and internationally: a report from a task force of the European Society for Clinical and Economic Aspects of Osteoporosis and Osteoarthritis (ESCEO). Semin Arthritis Rheum. 2014;44(3):253-263.

30. Chang KV, Hsiao MY, Chen WS, Wang TG, Chien KL. Effectiveness of intra-articular hyaluronic acid for ankle osteoarthritis treatment: a systematic review and meta-analysis. Arch Phys Med Rehabil. 2013;94(5):951-960. 
31. Witteveen AG, Sierevelt IN, Blankevoort L, Kerkhoffs GM, van Dijk $\mathrm{CN}$. Intra-articular sodium hyaluronate injections in the osteoarthritic ankle joint: effects, safety and dose dependency. Foot Ankle Surg. 2010;16(4):159-163.

32. Gómez-Aristizábal A, Kim KP, Viswanathan S. A systematic study of the effect of different molecular weights of hyaluronic acid on mesenchymal stromal cell-mediated immunomodulation. PLoS One. 2016;11(1): 0147868

33. Martel-Pelletier J. Pathophysiology of osteoarthritis. Osteoarthritis Cartilage. 2004;12:31-33.

34. Fioravanti A, Cantarini L, Chellini F, et al. Effect of hyaluronic acid (MW $500-730 \mathrm{kDa}$ ) on proteoglycan and nitric oxide production in human osteoarthritic chondrocyte cultures exposed to hydrostatic pressure. Osteoarthritis Cartilage. 2005;13(8):688-696.

35. Fukuda K, Dan H, Takayama M, Kumano F, Saitoh M, Tanaka S. Hyaluronic acid increases proteoglycan synthesis in bovine articular cartilage in the presence of interleukin-1. J Pharmacol Exp Ther. 1996;277(3):1672-1675.

36. Shimazu A, Jikko A, Iwamoto M, et al. Effects of hyaluronic acid on the release of proteoglycan from the cell matrix in rabbit chondrocyte cultures in the presence and absence of cytokines. Arthritis Rheum. 1993;36(2):247-253

37. Bolay H, Moskowitz MA. Mechanisms of pain modulation in chronic syndromes. Neurology. 2002;59(5 Suppl 2):S2-S7.

38. Latremoliere A, Woolf CJ. Central sensitization: a generator of pain hypersensitivity by central neural plasticity. J Pain. 2009;10(9):895-926.

39. Schuelert N, Mcdougall JJ. Grading of monosodium iodoacetate-induced osteoarthritis reveals a concentration-dependent sensitization of nociceptors in the knee joint of the rat. Neurosci Lett. 2009;465(2):184-188.

40. Ferreira-Gomes J, Adães S, Castro-Lopes JM. Assessment of movementevoked pain in osteoarthritis by the knee-bend and CatWalk tests: a clinically relevant study. J Pain. 2008;9(10):945-954.

41. Thakur M, Rahman W, Hobbs C, Dickenson AH, Bennett DL. Characterisation of a peripheral neuropathic component of the rat monoiodoacetate model of osteoarthritis. PLoS One. 2012;7(3):e33730.

42. Ikeuchi M, Izumi M, Aso K, Sugimura N, Kato T, Tani T. Effects of intra-articular hyaluronic acid injection on immunohistochemical characterization of joint afferents in a rat model of knee osteoarthritis. Eur J Pain. 2015;19(3):334-340.

43. Lin YT, Ro LS, Wang HL, Chen JC. Up-regulation of dorsal root ganglia BDNF and trkB receptor in inflammatory pain: an in vivo and in vitro study. J Neuroinflammation. 2011;8(1):126.
44. Havelin J, Imbert I, Cormier J, Allen J, Porreca F, King T. Central sensitization and neuropathic features of ongoing pain in a rat model of advanced osteoarthritis. J Pain. 2016;17(3):374-382.

45. Inoue K, Tsuda M. Microglia in neuropathic pain: cellular and molecular mechanisms and therapeutic potential. Nat Rev Neurosci. 2018;19(3):138-152.

46. Gallagher B, Tjoumakaris FP, Harwood MI, Good RP, Ciccotti MG, Freedman KB. Chondroprotection and the prevention of osteoarthritis progression of the knee. Am J Sports Med. 2015;43(3):734-744.

47. Park YS, Lim SW, Lee IH, Lee TJ, Kim JS, Han JS. Intra-articular injection of a nutritive mixture solution protects articular cartilage from osteoarthritic progression induced by anterior cruciate ligament transection in mature rabbits: a randomized controlled trial. Arthritis Res Ther. 2007;9(1):R8

48. Elmorsy S, Funakoshi T, Sasazawa F, Todoh M, Tadano S, Iwasaki N. Chondroprotective effects of high-molecular-weight cross-linked hyaluronic acid in a rabbit knee osteoarthritis model. Osteoarthritis Cartilage. 2014;22(1):121-127.

49. Altman RD, Manjoo A, Fierlinger A, Niazi F, Nicholls M. The mechanism of action for hyaluronic acid treatment in the osteoarthritic knee: a systematic review. BMC Musculoskelet Disord. 2015;16(1):321.

50. Vincent HK, Percival SS, Conrad BP, Seay AN, Montero C, Vincent KR. Hyaluronic acid (HA) viscosupplementation on synovial fluid inflammation in knee osteoarthritis: a pilot study. Open Orthop J. 2013;7(1):378-384.

51. Ishijima M, Nakamura $T$, Shimizu $K$, et al. Intra-articular hyaluronic acid injection versus oral non-steroidal anti-inflammatory drug for the treatment of knee osteoarthritis: a multi-center, randomized, open-label, non-inferiority trial. Arthritis Res Ther. 2014;16(1):R18.

52. Hendren L, Beeson P. A review of the differences between normal and osteoarthritis articular cartilage in human knee and ankle joints. Foot. 2009;19(3):171-176

53. Treppo S, Koepp H, Quan EC, Cole AA, Kuettner KE, Grodzinsky AJ. Comparison of biomechanical and biochemical properties of cartilage from human knee and ankle pairs. J Orthop Res. 2000;18(5): 739-748.

54. Kuettner KE, Cole AA. Cartilage degeneration in different human joints. Osteoarthritis Cartilage. 2005;13(2):93-103.

55. Eger W, Schumacher BL, Mollenhauer J, Kuettner KE, Cole AA. Human knee and ankle cartilage explants: catabolic differences. J Orthop Res. 2002;20(3):526-534.
Journal of Pain Research

\section{Publish your work in this journal}

The Journal of Pain Research is an international, peer reviewed, open access, online journal that welcomes laboratory and clinical findings in the fields of pain research and the prevention and management of pain. Original research, reviews, symposium reports, hypothesis formation and commentaries are all considered for publication.

\section{Dovepress}

The manuscript management system is completely online and includes a very quick and fair peer-review system, which is all easy to use. Visit http://www.dovepress.com/testimonials.php to read real quotes from published authors. 